\title{
Symbol Error Probability Analysis of a Multiuser Detector for $M$-PSK Signals Based on Successive Cancellation
}

\author{
Gerard J. M. Janssen, Member, IEEE, and Slimane Ben Slimane, Member, IEEE
}

\begin{abstract}
A narrow-band multiuser receiver based on successive signal detection and subtraction, is considered in this paper. The symbol error probability (SEP) for $M$-PSK modulated signals is evaluated and analytical approximations for the SEP of the individual signals are presented and compared with results obtained from simulations. For geometrically related signal amplitudes, a constant minimum distance can be guaranteed independent of the number of signals. The required amplitude ratio is shown to be related to $M$ and the number of co-channel signals. Optimizing the transmitted power for the different signals while ensuring the same SEP is then addressed and closed-form expressions of the signal amplitude ratios are derived. The effect of inaccurately estimated signal parameters due to noise is also analyzed. SEP results are presented for synchronous signals in an additive white Gaussian noise environment.
\end{abstract}

Index Terms-Multiuser detection, parameter estimation, signal cancellation, symbol error rate.

\section{INTRODUCTION}

A CHIEVING a high spectral efficiency is an important issue in future mobile communication systems. Increase of spectral efficiency allows for higher data rates or for more users to communicate simultaneously in the same bandwidth. In the third-generation mobile communication systems, code division multiple access (CDMA) is applied to achieve this goal. In CDMA, the user signal is coded with a user-signature which makes it possible to separate and detect users occupying the same channel simultaneously at the cost of a substantial increase in the bandwidth required. Recently, multiuser receiver techniques have been proposed for two narrow-band co-channel signals based on successive cancellation [2], or maximum-likelihood detection which has a much higher complexity [3] and [4].

In this paper, the symbol error probability (SEP) and the sensitivity to estimation errors of a narrow-band multiuser receiver for multiple $M$-PSK modulated signals, based on successive signal cancellation by means of subtraction, is investigated. Also power relations are derived which in principle allow

Manuscript received December 15, 2000; revised April 13, 2001 and June 6, 2001. This work was published in part in IEE Electronics Letters, vol. 36, no. 25, pp. 2103-2105, December 2000 [1]

G. J. M. Janssen is with the Telecommunication and Traffic-Control Systems Group, Dept. of Information Technology and Systems, Delft University of Technology, Delft, The Netherlands (e-mail: g.janssen@its.tudelft.nl).

S. Ben Slimane is with the Radio Communication Systems Group, Department of Signals, Sensors, and Systems, Royal Institute of Technology, Stockholm, Sweden (e-mail: slimane@radio.kth.se).

Publisher Item Identifier S 0733-8716(02)01003-X

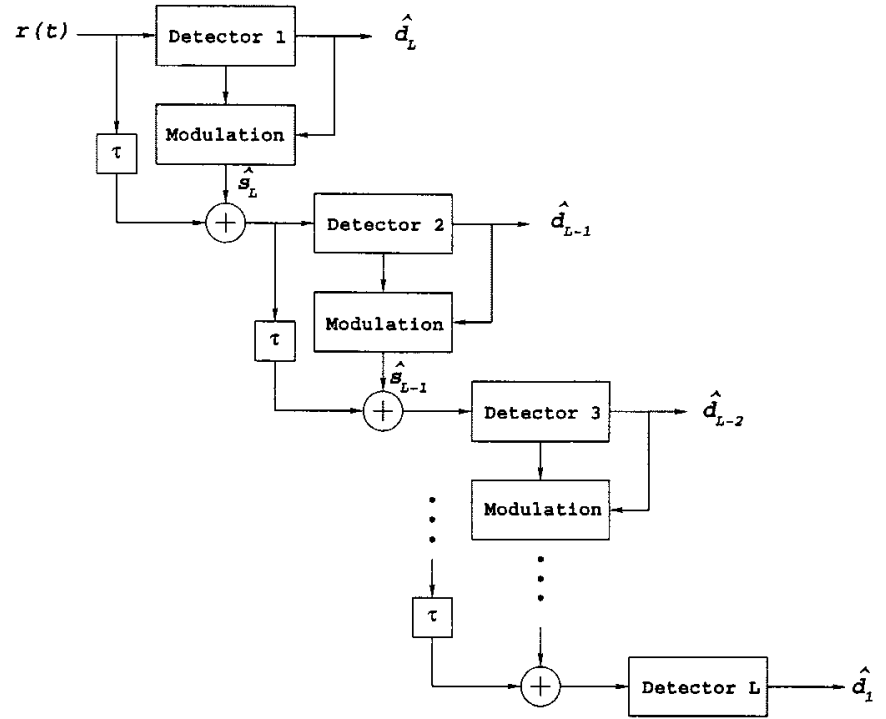

Fig. 1. Principle of the multiuser receiver using successive detection and subtraction.

assignment of an arbitrary number of co-channel signals for geometrically related signal powers. In the successive cancellation receiver, which is also applied as a multiuser detector for CDMA signals [5], the major signal is detected and estimated in the first detector; this estimate is subtracted from the total input signal. From this signal, subsequently the next largest signal is detected, estimated and subtracted, and so on. This principle is also known as "onion peeling" [6]. By narrowband, we refer to the fact that no spreading gain or bandwidth expanding signature code is applied to separate the users like in CDMA. The structure of the receiver is shown in Fig. 1.

For a single service system (e.g., speech service in GSM), the same link quality should be provided for all its connections, i.e., the average SEP should be the same for all users. An analytical method is presented to determine the received symbol energies for each of the $M$-PSK modulated co-channel signals which result in equal SEP for each of the signals for the multiuser detector described above. These results will be very useful when applying transmitter power control.

The signal state structure of multiple signals is investigated in Section II. In Section III, the SEP of the successive cancellation receiver is analyzed for $M$-PSK modulated signals and computationally less complex approximations are derived. SEP simulation results are presented for the Gaussian noise channel and compared with the proposed approximations. In Section IV, the required received power of the different signals is optimized 
while keeping their average SEP the same. In Section V, the effect of nonperfect parameter estimation due to the presence of noise is considered and analyzed. Conclusions are drawn in Section VI.

\section{Signal State Structure}

We consider a symbol-synchronous multiuser system with $L$ active users, all using the same carrier frequency and $M$-PSK modulation. With such a structure, the bandwidth efficiency $\rho$ is given by

$$
\rho=L \log _{2}(M) \quad \text { bits/s/Hz }
$$

which increases linearly with the number of users per channel. Denoting by $s_{i}(t)$ (Signal $i$ ) the transmitted signal of user $i$, the equivalent lowpass of the received signal sample at a given symbol interval $n T_{s}$ can be written as follows:

$$
r_{n}=\sum_{i=1}^{L} s_{n, i}+n_{n}=\sum_{i=1}^{L} \sqrt{E_{i}} e^{j\left(\theta_{i}+a_{n, i} \frac{2 \pi}{M}\right)}+n_{n}
$$

where $E_{i}$ is the symbol energy of user $i, a_{n, i}=0,1, \ldots,(M-$ 1) with equal probability of occurrence is related to the $n$th transmitted symbol of user $i$ and $n_{n}$ is the sampled additive white Gaussian noise (AWGN). The carrier phases $\theta_{i}$ are independent and uniformly distributed over $[0,2 \pi)$. We further assume that the signals have different energies with $E_{1}<E_{2}<$ $\cdots<E_{L}$.

Ignoring noise, the interference term in (2) will cause clouds around the major signal points due to the random phases and symbols. This is shown in Fig. 2 for four independent 4-PSK modulated signals where the receiver is locked to the major one.

The task of the first receiver is to detect the cloud belonging to the correct symbol value of the major signal. After correct cancellation of the major signal, the remaining signals will form clouds around the next major signal states, and so on. With $k$ signals present, the signal state regions are annular-shaped clouds which are limited by an inner and outer circle with radii

$$
\begin{aligned}
& R_{k, \text { min }}=\max \left(\sqrt{E_{k-1}}-\sum_{i=1}^{k-2} \sqrt{E_{i}}, 0\right) \text { and } \\
& R_{k, \text { max }}=\sum_{i=1}^{k-1} \sqrt{E_{i}}
\end{aligned}
$$

respectively. For $k=2$, the signal states are on a circle with radius $R_{2}=\sqrt{E_{1}}$ centered around the state of Signal 2 .

\section{SEP ANALYSIS}

\section{A. Conditional SEP}

Let us first consider the detection of Signal $k$. Assuming correct decisions for the first $L-k$ (strongest) signals, the coherent detector of $s_{k}(t)$ should be able to lock on its proper signal. In

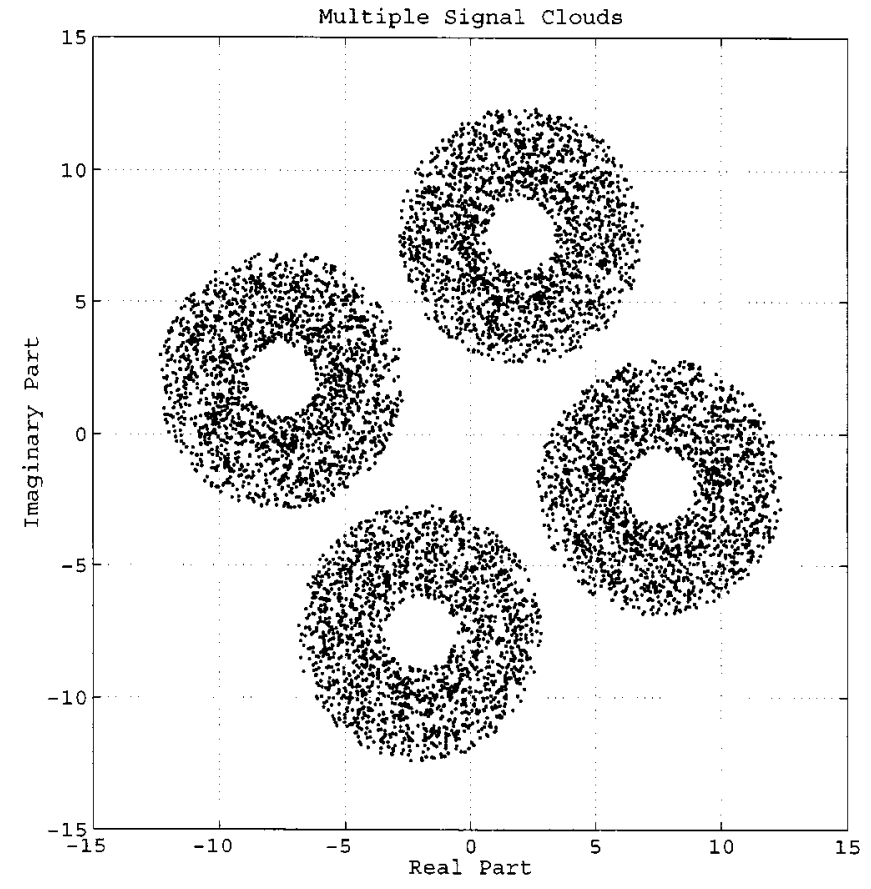

Fig. 2. Signal clouds around the dominant signal states with $\sqrt{E_{k}}=$ $2.5^{k-1}, k=1, \ldots, 4$, and $\theta_{4}=\pi / 6$ with random phases for the signals $s_{1}, \ldots, s_{3}$.

that, after phase compensation the received sample during the symbol interval $n T_{s}$ at the input of this detector (see Fig. 1) can be written as

$$
y_{n, k}=\sqrt{E_{k}} e^{j a_{n, k} \frac{2 \pi}{M}}+\sum_{i=1}^{k-1} \sqrt{E_{i}} e^{j \phi_{n, i}}+n_{n}
$$

where $\phi_{n, i}=\theta_{i}+a_{n, i}(2 \pi / M)-\theta_{k}$ is the total phase of Signal $i$ during the symbol interval $n T_{s}$, which can be considered uniformly distributed over $[0,2 \pi$ ). The conditional SEP (no symbol errors in the stronger signals detected in the $L-k$ stages) for $s_{k}(t)$ can be written as shown in (4), at the bottom of the page, for any $k \geq 2$ and

$$
P_{1}^{s} \approx \frac{a_{d}}{2} \operatorname{erfc}\left(\sqrt{\frac{E_{1} \sin ^{2}\left(\frac{\pi}{M}\right)}{N_{0}}}\right)
$$

for $k=1$. The parameter $a_{d}$ is related to the modulation level and is given by [7]

$$
a_{d}= \begin{cases}1, & \text { for BPSK } \\ 2, & \text { for higher modulation levels }(M>2) .\end{cases}
$$

Using (4), the value for $P_{k}^{s}$ can be solved numerically, however, the computational complexity increases with the number of signals. From the observation of Fig. 2, three approximations to the above conditional SEP can be obtained which will largely simplify its computation.

Approximation 1: Considering a worst case situation for the detection of $s_{k}(t)$ where all smaller signals are in-phase and ori-

$$
\begin{aligned}
P_{k}^{s} & =\operatorname{Pr}(\mathcal{E} \mid \text { no error in the } L-k \text { stages }) \\
& \approx \frac{a_{d}}{2(2 \pi)^{k-1}} \int_{0}^{2 \pi} \cdots \int_{0}^{2 \pi} \operatorname{erfc}\left[\frac{\sqrt{E_{k}} \sin \left(\frac{\pi}{M}\right)+\sum_{j=1}^{k-1} \sqrt{E_{j}} \cos \varphi_{j}}{\sqrt{N_{0}}}\right] d \varphi_{1} \ldots d \varphi_{k-1}
\end{aligned}
$$


ented such that the minimum distance to the decision threshold is achieved, the resulting conditional SEP results in an upper bound given by

$$
P_{k}^{s} \leq \frac{a_{d}}{2} \operatorname{erfc}\left(\frac{\sqrt{E_{k}} \sin (\pi / M)-R_{k, \max }}{\sqrt{N_{0}}}\right) .
$$

Approximation 2: A much tighter upper bound is found by considering that the in-phase smaller signals can be located anywhere on the outer circle of the annular region

$$
P_{k}^{s} \leq \frac{a_{d}}{4 \pi} \int_{0}^{2 \pi} \operatorname{erfc}\left(\frac{\sqrt{E_{k}} \sin \left(\frac{\pi}{M}\right)+R_{k, \max } \cos \theta}{\sqrt{N_{0}}}\right) d \theta .
$$

This upper bound becomes the exact conditional SEP for $k=2$.

Approximation 3: For $k>2$ all signal states can be assumed to be uniformly distributed over the annular region between $R_{k, \max }$ and $R_{k, \min }$. This results in an approximated conditional SEP having the form of

$$
\begin{aligned}
P_{k}^{s} \approx & \frac{a_{d}}{2 \pi\left(R_{k, \max }^{2}-R_{k, \text { min }}^{2}\right)} \\
& \times \int_{0}^{2 \pi} \int_{R_{k, \text { min }}}^{R_{k, \text { max }}} \operatorname{erfc}\left(\frac{\sqrt{E_{k}} \sin \left(\frac{\pi}{M}\right)+r \cos \theta}{\sqrt{N_{0}}}\right) r d r d \theta .
\end{aligned}
$$

For large values of $k$, this approximation is more accurate than Approximation 2 as discussed in Section III-C.

Example: As an illustrative example, let us assume that the amplitudes of the different signals are geometrically related (e.g., obtained by transmitter power control) with

$$
\sqrt{E_{k}}=\alpha^{k-1} \sqrt{E_{1}}, \quad \alpha>1 \quad \text { and } \quad 1 \leq k \leq L .
$$

The worst case conditional SEP of (6) becomes

$$
P_{k}^{s} \approx \frac{a_{d}}{2} \operatorname{erfc}\left(g(\alpha, M, k) \sqrt{\frac{E_{1}}{N_{0}}}\right)
$$

where the function $g(\alpha, M, k)$ is given by

$$
g(\alpha, M, k) \triangleq \alpha^{k-1} \sin \frac{\pi}{M}-\frac{\alpha^{k-1}-1}{\alpha-1} .
$$

$a_{d}$ is as earlier defined and $E_{1}$ is the average symbol energy of Signal 1. It is obvious from the above expression that the conditional SEP is dependent on the parameters $\alpha, M$, and the number of signals $k$.

To be able to detect $s_{k}(t)$ without inherent errors, the function $g(\alpha, M, k)$ has to be strictly positive. The function $g(\alpha, M, k)$ has the following interesting properties.

1) For $1<\alpha<1+1 / \sin (\pi / M)$, the function $g(\alpha, M, k)$ is a decreasing function of $k$ with maximum value $\sin (\pi / M)$ obtained at $k=1$. This puts a limit on the maximum number of signals that can be simultaneously received. Table I gives the value of $\alpha$ for which the function $g(\alpha, M, k)=0$ for different $k$ s and modulation levels $M$.

2) For $\alpha=1+1 / \sin (\pi / M)$, the function $g(\alpha, M, k)=$ $\sin (\pi / M), \forall k \geq 1$.
TABLE I

The Set of VAlues $(\alpha, k, M)$ FOR Which $g(\alpha, k, M)=0$

\begin{tabular}{c||c|c||c|c}
\hline \multicolumn{1}{c||}{ Number of Signals } & \multicolumn{2}{c||}{$M=2$} & \multicolumn{2}{c}{$M=4$} \\
\hline \hline$k$ & $\alpha$ & $\alpha[\mathrm{dB}]$ & $\alpha$ & $\alpha[\mathrm{dB}]$ \\
\hline 2 & 1 & 0 & 1.42 & 3.02 \\
\hline 3 & 1.62 & 4.19 & 2.09 & 6.40 \\
\hline 4 & 1.84 & 5.30 & 2.30 & 7.23 \\
\hline 5 & 1.93 & 5.71 & 2.37 & 7.49 \\
\hline 6 & 1.97 & 5.89 & 2.40 & 7.59 \\
\hline
\end{tabular}

3) For $\alpha>1+1 / \sin (\pi / M)$, the function $g(\alpha, M, k)$ is an increasing function of $k$ with minimum value $\sin (\pi / M)$ obtained at $k=1$. The number of signals, that can be simultaneously received in this case, is in principle not limited. However, the minimum required value of $\alpha$ increases with the modulation level $M$.

\section{B. Average SEP}

A symbol error at a given stage of the successive cancellation receiver will result in a partial suppression or even enhancement of the signal after subtraction which is translated into interference to the next stages. As the stronger signals are detected first, any error propagation will jam all remaining signals and will almost certainly result in symbol errors to these remaining signals.

Theorem 1: The average SEP of Signal $k$ of the narrow-band multiuser receiver which is based on successive signal detection and subtraction for $M$-PSK signals is given by

$$
\begin{aligned}
P_{k}= & \frac{M-1}{M}\left[1-\prod_{i=k+1}^{L}\left(1-P_{i}^{s}\right)\right] \\
& +P_{k}^{s} \prod_{i=k+1}^{L}\left(1-P_{i}^{s}\right), \quad 1 \leq k<L \\
P_{L}= & P_{L}^{s}
\end{aligned}
$$

where $M$ is the modulation level and $P_{k}^{s}$ is the conditional SEP of Signal $k$ as defined in (4) and (5).

Proof: Let us define the following two events:

$$
\begin{aligned}
& \mathcal{A}=\{\text { error occurred in the } L-k \text { stages }\} \\
& \mathcal{B}=\{\text { no error occurred in the } L-k \text { stages }\} .
\end{aligned}
$$

The average SEP of Signal $k$ is obtained as follows:

$$
P_{k}=\operatorname{Pr}(\mathcal{E} \mid \mathcal{A}) \operatorname{Pr}(\mathcal{A})+\operatorname{Pr}(\mathcal{E} \mid \mathcal{B}) \operatorname{Pr}(\mathcal{B}) .
$$

During the symbol interval $n T_{s}$, the input sample of detector $L-k+1$ can be written as

$$
y_{n, k}=s_{n, k}+\sum_{i=k+1}^{L}\left(s_{n, i}-\hat{s}_{n, i}\right)+\sum_{i=1}^{k-1} s_{n, i}+n_{n}
$$

where $\hat{s}_{n, i}$ is the estimate of $s_{n, i}$.

The conditional SEP can then be rewritten as follows:

$$
\begin{aligned}
\operatorname{Pr}(\mathcal{E} \mid \mathcal{B}) & =\operatorname{Pr}\left(\mathcal{E} \mid \hat{s}_{n, k+1}=s_{n, k+1}, \ldots, \hat{s}_{n, L}=s_{n, L}\right) \\
& =P_{k}^{s} .
\end{aligned}
$$


The probability of no error in the $L-k$ stages is obtained as

$$
\begin{aligned}
\operatorname{Pr}(\mathcal{B}) & =\operatorname{Pr}\left(\hat{s}_{n, k+1}=s_{n, k+1}, \ldots, \hat{s}_{n, L}=s_{n, L}\right) \\
& =\prod_{i=k+1}^{L}\left(1-P_{i}^{s}\right)
\end{aligned}
$$

which is only related to the conditional SEP since the input sample of any of those stages is free of interference from previous stages, i.e.,

$$
y_{n, l}=s_{n, l}+\sum_{i=1}^{l-1} s_{n, i}+n_{n}, \quad l>k .
$$

When a symbol error occurs at any of the $L-k$ stages, the remaining signals are jammed after signal subtraction. Thus, the detection of Signal $k$ will simply be based on a guess from $M$ possible symbols. That is

$$
\operatorname{Pr}(\mathcal{E} \mid \mathcal{A})=\frac{M-1}{M}
$$

Replacing (17), (18), and (20) in (15), (12) is obtained and the proof is complete.

We notice from Theorem 1 that only the strongest signal, $s_{L}(t)$, does not experience error propagation. An $M$-PSK symbol represents $N=\log _{2}(M)$ bits. An error caused by error propagation results in an error of the corresponding bits with probability 0.5 . If all larger signals were correctly detected, a symbol error results with high probability in one of the adjacent symbols and with an appropriate mapping scheme causes only a single bit error. Under these assumptions the bit error rate (BER) $P_{\mathrm{eb}, k}$ for $s_{k}(t)$ can be approximated by

$$
\begin{aligned}
P_{e b, k} \approx & \frac{1}{a_{d}} \frac{M-1}{M}\left[1-\prod_{i=k+1}^{L}\left(1-P_{i}^{s}\right)\right] \\
& +\frac{P_{k}^{s}}{N} \prod_{i=k+1}^{L}\left(1-P_{i}^{s}\right), \quad 1 \leq k<L \\
P_{\mathrm{eb}, L} \approx & \frac{P_{L}^{s}}{N} .
\end{aligned}
$$

\section{Simulation Results}

We compared average SEP results based on the three approximations, as derived in this section against SEP results obtained from simulations for 4-PSK modulated signals. The results are generated under the assumption that amplitude and phase are accurately known at the receiver for all signals, i.e., in case of a correct decision the signal is completely removed.

In Fig. 3, the SEP results from simulations and approximations are given as a function of $E_{1} / N_{0}$ for the case of four 4-PSK signals with $\alpha=1+1 / \sin (\pi / 4) \approx 2.41$. It is observed that Approximation 1 is good for Signal 1 but is rather loose for the other signals. It is also observed that all signals perform better than the minor signal. Approximation 2 is a much better upper bound for the SEP, yet still a little loose for the larger signals because all signal states are taken at $R_{k, \max }$.

The results from Approximation 3 are very close to the simulation results. Note that Approximation 3 is not a bound. For Signal 1 and Signal 2, the approximation is nearly exact. For Signal 3, the calculated SEP becomes slightly optimistic com-
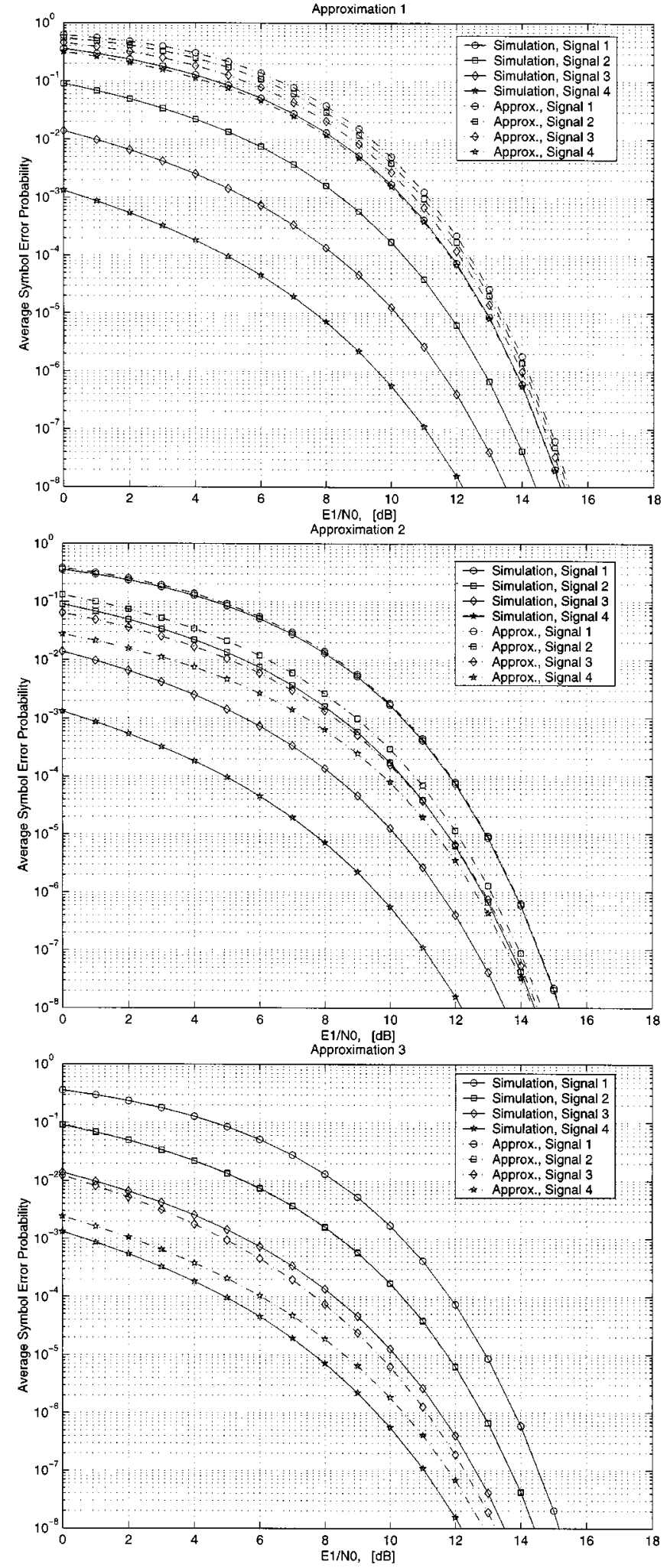

Fig. 3. Average SEP for 4-PSK modulation with $L=4$ and $\alpha=2.41$. Performance comparison between the different approximations and simulation results.

pared with that of Signal 4 which is slightly too large. This error occurs because we assume uniformly distributed signal states over the annular region, whereas in reality the density of the signal states is larger at the outside of the annular region than at the inside for Signal 3, the opposite occurs for Signal 4. 


\section{OPTIMIZATION OF SigNAL POWERS}

We have seen in the previous sections that it is possible to transmit multiple narrow-band signals over the same bandwidth and be able to detect them reliably. It has been shown that the performance is dependent on the relation between the received powers of the different signals. It is, therefore, important to control the transmitted power for every signal to ensure the required quality of service, which requires a feedback channel from the receiver to the transmitter. A good system should provide the same link quality for all its connections of the same service type. Thus, for a multiuser system with single service the best choice of the parameters $E_{k} \mathrm{~s}$ is such that the average SEP is the same for all the different users. That is

$$
P_{1}=P_{2}=\cdots=P_{L}=P_{\text {sep }} .
$$

The above condition ensures the same SEP and minimum received power for the $L$ different links.

Theorem 2: The conditional SEP that ensures the same average SEP for all the different $M$-PSK modulated signals of the multiuser receiver with successive signal detection and signal subtraction is given by

$$
\begin{aligned}
P_{k}^{s} & =\operatorname{Pr}(\mathcal{E} \mid \mathcal{B}) \\
& =\frac{\left(1-\frac{1}{M}\right) \frac{1}{M^{L-k}} P_{\text {sep }}}{1-\frac{1}{M}-\left(1-\frac{1}{M^{L-k}}\right) P_{\text {sep }}}, \quad 1 \leq k \leq L
\end{aligned}
$$

where $P_{\text {sep }}$ is the preset average SEP required for the different links, $\mathcal{B}$ is the error event defined in (14), and $M$ is the modulation level.

Proof: From (12) and the condition of (23), the conditional SEP is obtained as

$$
P_{k}^{s}=\frac{P_{\mathrm{sep}}-1+\frac{1}{M}}{\prod_{i=k+1}^{L}\left(1-P_{i}^{s}\right)}+1-\frac{1}{M}, \quad 1 \leq k \leq L .
$$

Using the above expression, we can form the following ratio:

$$
\frac{P_{k}^{s}-1+\frac{1}{M}}{P_{k-1}^{s}-1+\frac{1}{M}}=1-P_{k}^{s}
$$

which can be further simplified giving a simple recursive relation

$$
P_{k-1}^{s}=\frac{1}{M} \frac{P_{k}^{s}}{1-P_{k}^{s}}
$$

Using the above recursion the expression of the conditional SEP can be proven by induction.

The conditional SEP is illustrated in Fig. 4 as a function of the preset SEP when $L=4$ signals. We notice that this error probability is a linear function of $P_{\text {sep }}$ over the error probability range of interest $\left(P_{\text {sep }} \leq 10^{-1}\right)$ and (24) can be approximated by

$$
P_{k}^{s} \approx \frac{1}{M^{L-k}} P_{\text {sep }}
$$

which becomes exact when $k=L$.

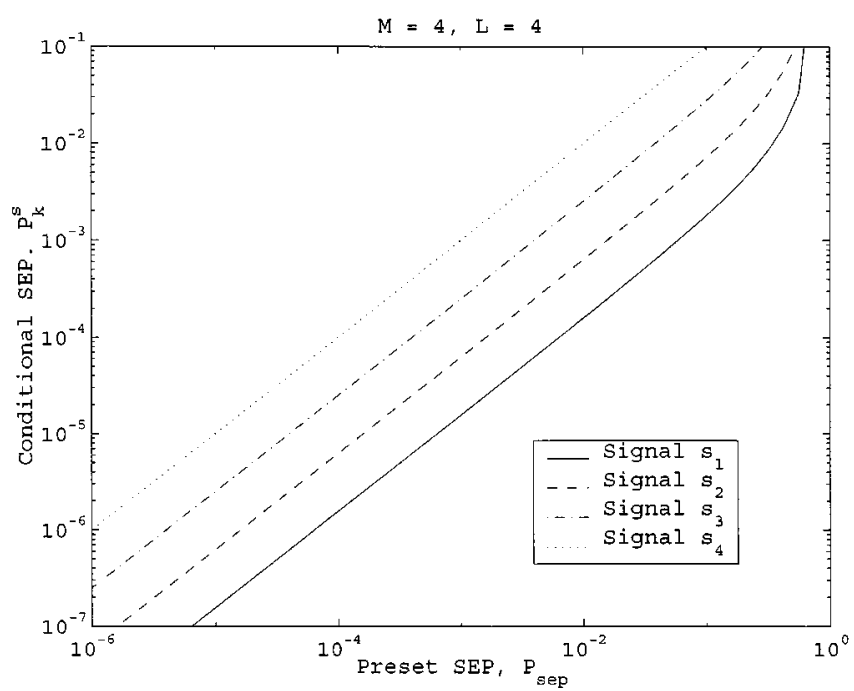

Fig. 4. Conditional SEP as a function of the preset error probability for the case of four 4-PSK signals in AWGN channels.

Combining (5) and (24) with $k=1$, the preset SEP can be written as a function of the signal-to-noise ratio (SNR) of Signal 1 as

$$
P_{\text {sep }} \approx \frac{\frac{a_{d}}{2} \operatorname{erfc}\left[\sqrt{\frac{E_{1}}{N_{0}}} \sin \left(\frac{\pi}{M}\right)\right]}{\frac{1}{M^{L-1}}+\frac{1-\frac{1}{M^{L-1}}}{1-\frac{1}{M}} \frac{a_{d}}{2} \operatorname{erfc}\left[\sqrt{\frac{E_{1}}{N_{0}}} \sin \left(\frac{\pi}{M}\right)\right]} .
$$

This error probability is illustrated in Fig. 5 as a function of $E_{1} / N_{0}$ and for different number of users, $L$. We notice that for a given preset SEP, the required SNR for the weakest signal increases with $L$. This is natural because when increasing the number of users, the propagation error toward the weakest signal increases and a higher SNR is then needed to compensate for that. Surprisingly, this increase in SNR is quite small. For instance, at a preset error probability of $10^{-4}$ only $1-\mathrm{dB}$ increase in $E_{1} / N_{0}$ to go from a single user per channel to $L=4$ users per channel and a 2-dB increase to support eight users per channel.

Given the required SEP of the system (usually determined by the type of service offered), Fig. 5 can be used to get the SNR needed for Signal 1. The next step is to determine the required powers for the remaining $L-1$ signals. The objective is to find the minimum power needed for every signal such that their average SEPs are the same. This will not only reduce the transmitted power of the different users but also simplify the performance evaluation of the system since the same SEP curve can be used for the $L$ different users.

The required powers of the different signals can be determined from (24) together with one of the expressions of $P_{k}^{s}$ derived in Section III. Two approximations that result in closed-form solutions for the signal powers are considered in Sections IV-A-C.

\section{A. The Gaussian Approximation}

In the first approximation, we assume that when detecting $s_{k}(t)$, the linear combination of the smaller-not yet detected-signals $s_{k-1}(t), \ldots, s_{1}(t)$ can be modeled as a 


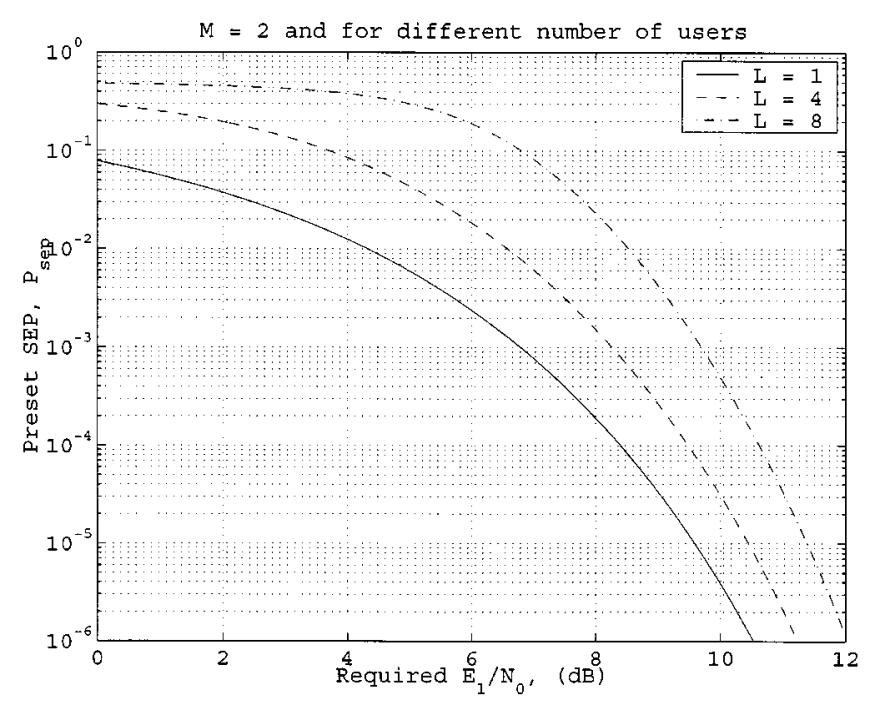

Fig. 5. The required SNR for BPSK modulation of the weakest signal $s_{1}$ for a given preset SEP and for different number of users.

Gaussian distributed zero-mean random variable with a variance equal to the sum of their energies. The conditional SEP from (4) then becomes

$$
P_{k}^{s}=\frac{a_{d}}{2} \operatorname{erfc}\left(\sqrt{\frac{E_{k} \sin ^{2}\left(\frac{\pi}{M}\right)}{\sum_{i=1}^{k-1} E_{i}+N_{0}}}\right), \quad 1 \leq k \leq L .
$$

Combining (29) with (24) and solving for the average symbol energy $E_{k}$ we get

$E_{k}= \begin{cases}N_{0} \frac{F^{2}\left(P_{\mathrm{sep}}, M, 1\right)}{\sin ^{2}\left(\frac{\pi}{M}\right)}, & k=1 \\ E_{k-1}\left[\frac{F^{2}\left(P_{\mathrm{scp}}, M, k\right)}{\sin ^{2}\left(\frac{\pi}{M}\right)}+\frac{F^{2}\left(P_{\mathrm{sep}}, M, k\right)}{F^{2}\left(P_{\mathrm{sep}}, M, k-1\right)}\right], & 2 \leq k \leq L\end{cases}$

where

$F\left(P_{\mathrm{sep}}, M, k\right)=\operatorname{erf}^{-1}\left[1-\frac{2}{a_{d}} \frac{\left(1-\frac{1}{M}\right) \frac{1}{M^{L-k}} P_{\mathrm{sep}}}{\frac{1}{M}-\left(1-\frac{1}{M^{L-k}}\right) P_{\mathrm{sep}}}\right]$.

Given the average symbol energy of the smaller signal, the required average energy of the next stronger signal can then be obtained. The signal energies of the stronger signals can also be written as a function of the symbol energy $E_{1}$ as follows:

$$
E_{k}=\alpha_{\mathrm{gap}, k}^{2} E_{1}
$$

with

$$
\alpha_{\text {gap }, k}^{2}=\prod_{i=2}^{k}\left[\frac{F^{2}\left(P_{\text {sep }}, M, i\right)}{\sin ^{2}\left(\frac{\pi}{M}\right)}+\frac{F^{2}\left(P_{\text {sep }}, M, i\right)}{F^{2}\left(P_{\text {sep }}, M, i-1\right)}\right]
$$

which is a function of the preset SEP $P_{\text {sep }}$ and the modulation level $M$.

Using the relation between the average energy per symbol and the average signal power we can write

$$
\text { Pow }_{k}=\frac{E_{k}}{T_{s}}=\alpha_{\text {gap }, k}^{2} \text { Pow }_{1}
$$

where $T_{s}$ is the symbol duration and Pow $_{k}$ is the power of user $k$. Thus, the coefficient $\alpha_{\text {gap }, k}^{2}$ can be seen as the required power margin between $s_{k}(t)$ and $s_{1}(t)$ that ensures the same average SEP for the different signals.

\section{B. Minimum Distance Approximation (MDA)}

In the second case we approximate the conditional SEP $P_{k}^{s}$ by Approximation 1 given in (6) and introduced in Section III. This approximation assumes that the resulting signal state is at the minimum distance to the decision threshold and occurs when all smaller signals are in-phase and oriented toward one of the neighboring signal states. Combining (6) with (24) and solving for the average symbol energy $E_{k}$ we get

$$
\sqrt{E_{k}}= \begin{cases}\sqrt{N_{0}} \frac{F\left(P_{\mathrm{scp}}, M, 1\right)}{\sin \left(\frac{\pi}{M}\right)}, & k=1 \\ \gamma \sqrt{E_{k-1}}+\frac{\sqrt{N_{0}} F\left(P_{\mathrm{scp}}, M, 1\right)}{\sin \left(\frac{\pi}{M}\right)} \beta_{k}, & 2 \leq k \leq L\end{cases}
$$

where

$$
\begin{aligned}
\gamma & =1+\frac{1}{\sin \left(\frac{\pi}{M}\right)} \\
\beta_{k} & =\frac{\left[F\left(P_{\mathrm{sep}}, M, k\right)-F\left(P_{\mathrm{sep}}, M, k-1\right)\right]}{F\left(P_{\mathrm{sep}}, M, 1\right)}
\end{aligned}
$$

and $F\left(P_{\text {sep }}, M, k\right)$ is as earlier defined in (30).

Combining the two expressions of (34), the average symbol energy $E_{k}$, becomes

$$
\sqrt{E_{k}}=\gamma \sqrt{E_{k-1}}+\beta_{k} \sqrt{E_{1}}, \quad k=2, \ldots, L .
$$

After some manipulations, the average symbol energy of Signal $k$ can be expressed as a function of the symbol energy of Signal 1 as

$$
\begin{aligned}
E_{k} & =\alpha_{\text {mdap }, k}^{2} E_{1} \\
& =\gamma^{2(k-1)}\left(1+\sum_{i=1}^{k-1} \frac{\beta_{i+1}}{\gamma^{i}}\right)^{2} E_{1} .
\end{aligned}
$$

All signal energies of the stronger signals are now obtained from that of Signal 1. The coefficient relating these signal energies is dependent on the SEP $P_{\text {sep }}$ and the modulation level $M$.

\section{Results}

The required power margins between the different narrow-band signals for equal SEP performance are computed and plotted as a function of the preset SEP based on both the Gaussian approximation and the MDA. These power margins are then used to compute the actual achieved average SEP and compared with the preset SEP. The objective is of course to have the average SEP of all the signals equal to the preset SEP.

Fig. 6 illustrates the required power margins of the different signals with respect to that of Signal 1 to achieve the same SEP over an AWGN channel for the case of $L=4$ signals and the BPSK modulation technique. Both approximations as defined in (31) and (38) are given in this figure. It is observed that, for the case of the Gaussian approximation, the required power margin 


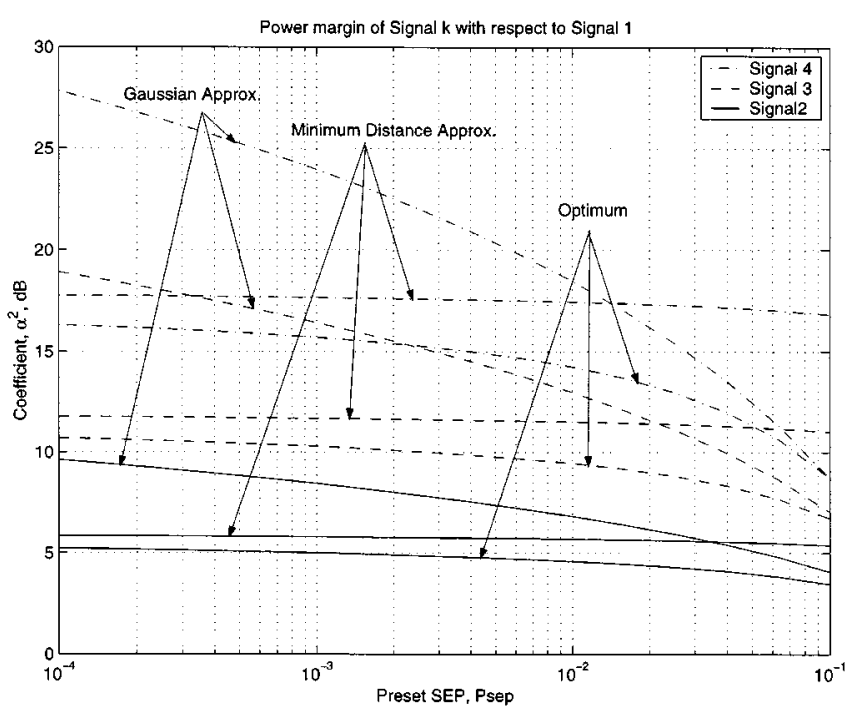

Fig. 6. Power margins of the stronger narrow-band signals with respect to the weakest signal as a function of the preset SEP.

between the different signals increases when the preset SEP decreases. To better understand this behavior and its relation to the system parameters, we can take a closer look to the expression of the power margin $\alpha_{\text {gap }, k}^{2}$ given in (31). At low preset SEP (high SNR) the conditional SEP takes the form of (27) and the power margin for the Gaussian approximation can be be simplified to ${ }^{1}$

$$
\alpha_{\text {gap }, k}^{2} \approx\left[1-\frac{\ln \left(P_{\text {sep }}\right)}{\sin ^{2}(\pi / M)}\right]^{k-1}, \quad P_{\text {sep }} \ll 1
$$

which is independent of the number of users $L$ but depends on the preset SEP, the modulation level $M$, and the signal order $k$.

For the case of the MDA, this power margin is almost constant and hardly changes with the preset SEP. Infact, at low preset SEP the coefficient $\beta_{k}$ of (36) converges to zero and the power margin for the MDA, given in (38), becomes

$$
\alpha_{\text {mdap }, k}^{2} \approx\left[1+\frac{1}{\sin (\pi / M)}\right]^{2(k-1)}, \quad P_{\text {sep }} \ll 1
$$

which is also independent of the total number of users $L$ but depends only on the modulation level $M$ and the signal order $k$. Note that, for high SNR, the power margin for the MDA is independent of the preset SEP while that for the Gaussian approximation increases when decreasing the preset SEP.

In Fig. 7, the average bit-error probabilities (BEPs) of the different signals are given as a function of the SNR, $E_{1} / N_{0}$, when the Gaussian approximation for the power margins is used. It is observed that the BEP of the different signals are similar and quite close to the preset SEP for low SNRs $\left(E_{1} / N_{0} \leq 6 \mathrm{~dB}\right)$. However, at high SNRs, the power levels are overestimated by this method making the BERs deviate from the preset SEP considerably.

\footnotetext{
${ }^{1}$ This simplification is obtained by using the approximation $\operatorname{erf}(x) \approx 1-$ $e^{-x^{2}}$ in (30) and then combining the result with (32).
}

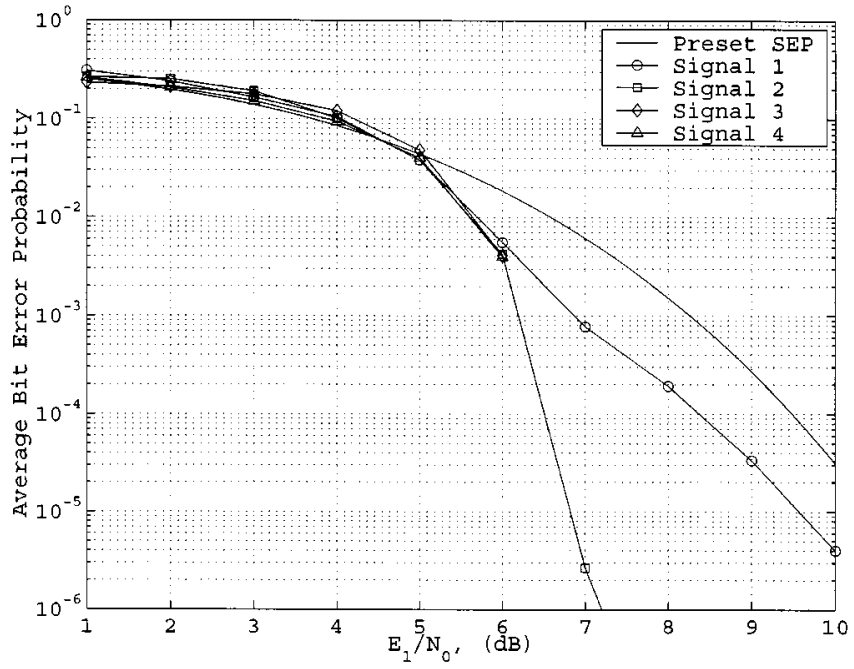

Fig. 7. Average BEPs of the narrow-band different signals as a function of $E_{1} / N_{0}$ in AWGN channels when the Gaussian approximation is used.

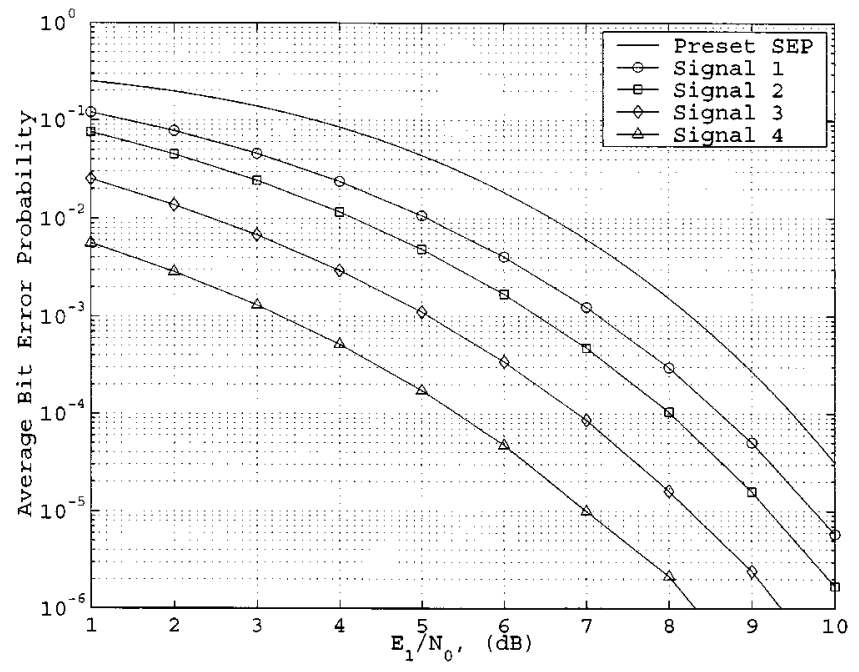

Fig. 8. Average BEPs of the different narrow-band signals as a function of $E_{1} / N_{0}$ in AWGN channels when the MDA is used.

The average BEPs of the different signals, when the MDA is used, are illustrated in Fig. 8 as a function of $E_{1} / N_{0}$. We notice here that this approximation overestimates the required power margins of the stronger signals making their average BERs lower than the preset error probability over all the SNR range. It is observed from this figure that these power margins are quite high for low SNRs and get smaller as the SNR increases. Thus, the MDA is better than the Gaussian approximation for high SNRs while the Gaussian approximation is preferred at low SNRs.

\section{EFFECT OF InACCURATE PARAMETER Estimation}

Accurate parameter estimation is crucial for the operation of the narrow-band multiuser detector based on successive signal cancellation. In practice, parameter estimation is not perfect due to the presence of noise and other co-channel signals.

We assume that the user signal is transmitted in short bursts with $N_{b}$ symbols per burst, part of which is a training sequence of $K\left(K<N_{b}\right)$ consecutive symbols. We further assume that 
the training sequences, denoted $\left\{w_{k}(t)\right\}$, of the different users are orthogonal with

$$
\int_{0}^{K T_{s}} w_{i}(t) w_{k}(t) d t=\left\{\begin{array}{ll}
K, & i=k \\
0, & i \neq k
\end{array} .\right.
$$

Thus, for synchronous multiuser systems, the inaccuracy in parameter estimations will be caused only by additive noise. Note that nonorthogonal training sequences and/or nonperfect synchronization between user signals will introduce extra errors in the signal parameter estimations. However, as shown in [8], with proper signal processing these extra errors can be reduced considerably. The results there showed that by using training sequences with low correlation, signals can be separated almost completely by applying the bootstrapping technique.

The equivalent lowpass of the received signal, containing the training sequences of the different signals, during a particular burst can be written as follows:

$$
\begin{array}{r}
r(t)=\sum_{k=1}^{L} w_{k}(t) \sqrt{E_{k}} e^{j \theta_{k}}+n_{I}(t)+j n_{Q}(t), \\
0 \leq t \leq K T_{s}
\end{array}
$$

where $w_{k}(t)$ is the transmitted training sequence of Signal $k, E_{k}$ is the symbol energy, and $\theta_{k}$ is a random phase assumed constant during the burst duration. $n_{I}(t)$ and $n_{Q}(t)$ are independent zero-mean Gaussian noise processes with equal spectral density $N_{0}$, respectively.

The signal parameters, $E_{k}$ and $\theta_{k}$, are estimated using the $K$ symbols of the training sequence as follows:

$$
\frac{1}{K} \int_{0}^{K T_{s}} w_{k}(t) r(t) d t=\sqrt{E_{k}} e^{j \theta_{k}}+\tilde{n}_{I, k}+j \tilde{n}_{Q, k} .
$$

By averaging over the $K$ symbols of the training sequence, the effect of noise is reduced by a factor of $K$. In that, the noise components, $\tilde{n}_{I, k}$ and $\tilde{n}_{Q, k}$, are independent Gaussian random variables with zero-mean and variance $N_{0} /(2 K)$.

Due to this imperfect parameter estimations, a residual interference will remain after signal subtraction even when the subtracted signals were correctly detected. For instance, with correct detection of the $L-k$ stronger signals, the input signal to detector $(L-k+1)$ during the symbol interval $n T_{s}$ can be written as

$$
\begin{aligned}
y_{n, k} & =s_{n, k}+\sum_{i=1}^{k-1} s_{n, i}+\sum_{i=k+1}^{L}\left(s_{n, i}-\hat{s}_{n, i}\right)+n_{n} \\
& =s_{n, k}+\sum_{i=1}^{k-1} s_{n, i}+z_{n, k}
\end{aligned}
$$

where

$$
z_{n, k}=\sum_{i=k+1}^{L} e^{j a_{n, k} \frac{2 \pi}{M}}\left(\tilde{n}_{I, k}+j \tilde{n}_{Q, k}\right)+n_{n}
$$

with $a_{n, k}$ as defined in (2).

Since the training sequences are orthogonal, the terms in the above summation are independent Gaussian random variables
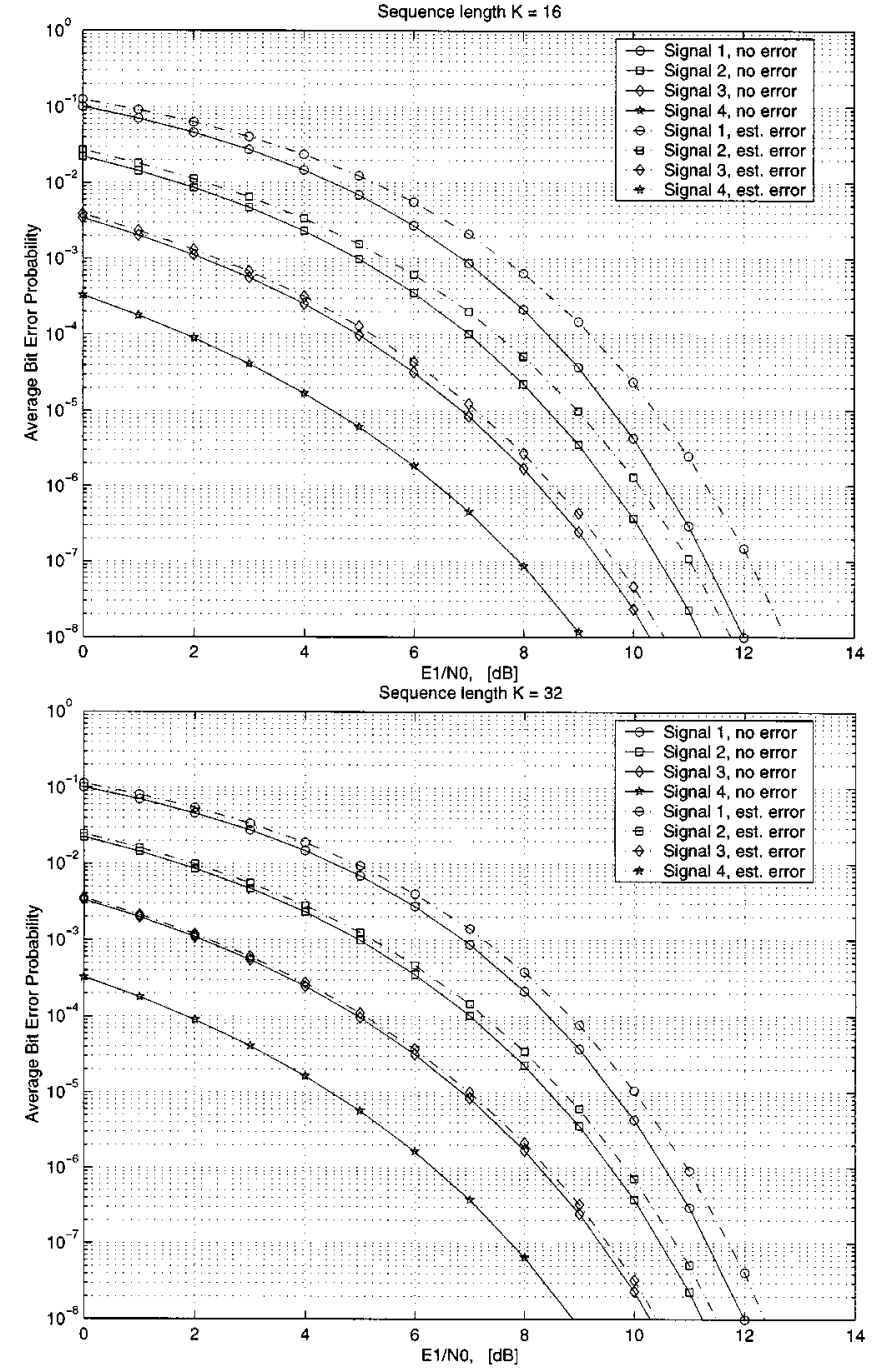

Fig. 9. Average BER for $L=4$ users with BPSK modulation and training sequences of length 16 and 32 , respectively.

[9], [10]. The sample $z_{n, k}$ is then a white Gaussian random variable with zero-mean and variance $[1+(L-k) / K] N_{0} / 2$. Thus, with imperfect parameter estimation, the conditional SEP takes the form of (4) with $N_{0}$ replaced by $[1+(L-k) / K] N_{0}$. The degradation increases for each successive signal to be detected and is largest for the minor signal (Signal 1). If the bandwidth of the estimation circuit is much smaller than the matched-filter bandwidth of the detector $(K \gg 1)$ then this degradation can be neglected. Fig. 9 shows the effect of estimation errors on the average BEP for training sequences of length $K=16$ and $K=32$ and $L=4$ signals with BPSK modulation. It is observed that the degradation for the minor signal (worst case) is relatively small. This degradation, due to estimation errors, can be compensated for by a small increase in SNR.

\section{CONCLUSION}

In this paper, we analyzed the SEP performance of a multiuser receiver for narrow-band $M$-PSK modulated signals, based on successive signal cancellation by means of subtraction. For a multisignal environment, three approximations for the conditional SEP were derived and compared with simulation results. 
It has been shown that for signals with geometrically related amplitudes, two important cases occur. If the amplitude relation between successive signals (ordered in amplitude) at the receiver $\alpha \geq 1+1 / \sin (\pi / M)$, in principle an arbitrary number of signals can be stacked. In practice, however, where signal parameter estimation is inaccurate, this will not be feasible. For $\alpha<1+1 / \sin (\pi / M)$, inherent errors occur if too many signals are stacked; the maximum number of signals $L$ decreases with decreasing $\alpha$ and with increasing $M$.

An analytical method is presented to determine the power margins at the receiver input for multiple co-channel signals in order to obtain equal SEP for each of the signals. The required power margins between the different signals for an AWGN channel are evaluated based on two approximations: the Gaussian approximation of the undetected signals and the MDA. It was found that the power margins derived with the Gaussian approximation are quite accurate for low SNRs but are much larger (several decibels) than required for high SNRs. The power margins derived with the MDA method are larger than needed at small SNR values but give better results at large SNR values.

Accurate parameter estimation is very important for the proposed multiuser detector. It has been shown that parameter estimation errors due to noise can be modeled as a small decrease in the SNR after each cancellation. This degradation can be reduced by increasing the length of the training sequence, or compensated for by adding an extra SNR margin.

\section{REFERENCES}

[1] G. J. M. Janssen and S. Ben Slimane, "Symbol error probability of a $M$-PSK multiuser detector using successive cancellation," Electron. Lett., vol. 36, pp. 2103-2105, Dec. 2000.

[2] M. Moretti, G. J. M. Janssen, and R. Prasad, "Performance evaluation of the dual signal receiver for a quadrature modulation scheme," in Proc. IEEE Globecom'98, Sydney, NSW, Australia, Nov. 1998, pp. 3542-3547.

[3] B. Hagerman, "Single-user receivers for partly known interference in multiuser environments," Ph.D. Dissertation, Radio Commun. Sys. Lab., Royal Inst. of Technol., Stockholm, Sweden, Oct. 1995.

[4] S. J. Grant and J. K. Cavers, "Performance enhancement through joint estimation of co-channel signal using diversity arrays," IEEE Trans. Commun., vol. 46, pp. 1039-1049, Aug. 1998.
[5] J. M. Holtzman, "DS/CDMA successive interference cancellation," in Proc. IEEE Int. Symp. Spread Spectrum Techniques and Applications, IEEE ISSSTA'94, Finland, 1994, pp. 69-78.

[6] T. M. Cover and J. A. Thomas, Elements of Information Theory. New York: Wiley, 1991.

[7] J. G. Proakis, Digital Communications. New York: McGraw-Hill, 1995.

[8] M. Moretti, E. Nostrato, S. Piagneri, and G. J. M. Janssen, "A frequency estimation scheme for a two-signal environment," in Proc. IEEE VTC'99, Amsterdam, The Netherlands, Sept. 1999, pp. 1800-1804.

[9] H. L. van Trees, Detection Estimation and Modulation Theory. New York: Wiley, 1968, pt. I.

[10] S. M. Kay, Fundamentals of Statistical Signal Processing. Englewood Cliffs, NJ: Prentice-Hall, 1993.

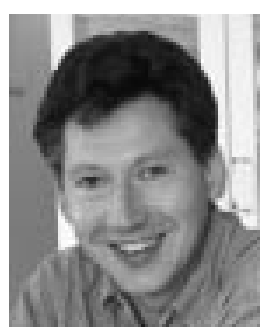

Gerard J. M. Janssen (M'93) received the M.Sc.E.E. degree from Eindhoven University of Technology in 1986, and the Ph.D. degree from Delft University of Technology, in 1998, both in the Netherlands.

In 1986, he joined the Physics and Electronics Laboratory of the Dutch Organization of Applied Scientific Research (TNO) where he was involved in radarcross-section modeling, radio direction finding, interference cancellation and wideband propagation measurements. He is currently an Associate Professor in the Telecommunications and Traffic Control Systems group at Delft University of Technology. His research interests are in wireless communication, especially narrow-band multiuser detection, modulation techniques, indoor radio propagation, and diversity techniques.

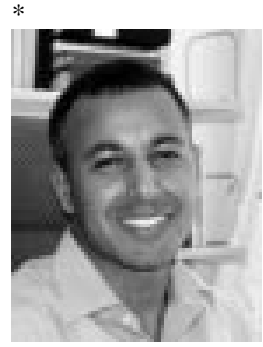

Slimane Ben Slimane (S'92-M'93) received the B.Sc. degree in electrical engineering from University of Quebec at Trois-Rivieres, Canada, in 1985, and the M.Sc. and Ph.D. degrees from Concordia University, Montreal, in 1988 and 1993, respectively.

During 1993-1995, he worked as a Research Associate and part-time Instructor at Concordia University. He is currently an Associate Professor in the Department of Signals, Sensors, and Systems, Royal Institute of Technlogy (KTH), Stockholm, Sweden. His research interest includes wireless communications with special emphasis on digital communication techniques for fading channels, error control coding, spread spectrum communications, and multicarrier transmission techniques. 